\title{
The Effect of Taurine on Plasma Cholesterol Concentration in Genetic Type 2 Diabetic GK Rats
}

\author{
Naomichi NishimurA*, Chie UMEDA, Hiroaki ODA ${ }^{1}$ \\ and Hidehiko YoKogosHI \\ School of Food and Nutritional Sciences, The University of Shizuoka, \\ 52-1 Yada, Shizuoka 422-8526, Japan \\ ${ }^{1}$ Department of Applied Biological Sciences, Nagoya University, Nagoya 464-8601, Japan
}

(Received May 15, 2002)

\begin{abstract}
Summary The purpose of this study is to investigate the effect of taurine on the plasma cholesterol concentration in genetic type 2 diabetic rats fed cholesterol-free or high-cholesterol diets. Diabetic rats (GK male rats) and normal rats (Wistar male rats) were fed either a cholesterol-free or cholesterol-enriched ( $1 \%$ cholesterol $+0.25 \%$ sodium cholate) diet supplemented with or without $3 \%$ taurine for 21 or $14 \mathrm{~d}$. Compared to the normal rats, diabetic rats showed a high glucose concentration in their blood and plasma, but it was not affected by taurine feeding. The plasma insulin concentration was higher in the diabetic rats than in the normal rats. At the start of the experiment, the plasma cholesterol concentration was significantly higher in the diabetic rats than in the normal rats. Taurine did not affect the plasma cholesterol level in rats fed the cholesterol-free diet. However, taurine feeding signifcantly increased the plasma HDL-cholesterol concentration in the diabetic rats fed the cholesterol-free diet. In both the diabetic and normal rats fed the cholesterol diet, the plasma cholesterol concentration was significantly lower in rats fed the diet supplemented with taurine than in the rats fed the control diet. It was concluded that taurine has a hypocholesterolemic effect in both diabetic and normal rats fed diets containing cholesterol. Moreover, these results suggest that taurine seems to affect the HDL-cholesterol metabolism in diabetic rats fed a cholesterol-free diet.
\end{abstract}

Key Words taurine, cholesterol, type 2 diabetes mellitus, rat, hypocholesterolemic effect

It is well known that atherosclerosis is induced as a complication of diabetes mellitus (1). This atherosclerosis is resultant of an abnormal lipid metabolism caused by diabetes mellitus, especially both elevated triglycerides (2) and reduced HDL in the serum (3). It is conceivable that alternation of the lipid metabolism in diabetes mellitus is responsible for insulin resistance or abnormal insulin secretion. The activity of lipoprotein lipase seems to be suppressed by reduced insulin secretion (4). Therefore, the metabolisms of cholesterol and bile acid in diabetes mellitus may be different from those in humans without diabetes mellitus. Ameliorating the abnormal cholesterol metabolism would be useful for preventing complications due to diabetes mellitus.

We found that taurine, 2-aminoethanesulfonic acid, reduced the VLDL-cholesterol in serum through the induction of cholesterol $7 \alpha$-hydroxylase (CYP7A1), the rate-limiting enzyme in the pathway to degrade cholesterol into bile acid, in normal rats fed cholesterol (5). Moreover, taurine elevated the HDL-cholesterol in normal rats fed a cholesterol-free $\operatorname{diet}(6)$.

* To whom correspondence should be addressed at present address: Department of Human Life and Development, Nayoro City College, Kita-8-1, Nishi-2, Nayoro, Hokkaido 096-8641, Japan

E-mail: nishimura@nayoro.ac.jp
In our previous study, taurine affected the cholesterol metabolism in streptozotocin (STZ)-induced diabetic rats (insulin-dependent diabetes mellitus model) fed diets supplemented with or without cholesterol $(7,8)$. In STZ-induced diabetic rats, insulin is not secreted from the pancreatic $\beta$-cells. However, few studies have examined the effect of taurine on the cholesterol metabolism in animals with type 2 diabetes mellitus (non-insulin-dependent diabetes mellitus (NIDDM)), which are resistant to insulin. Therefore, investigating changes in the cholesterol metabolism induced by taurine feeding in type 2 diabetic rats is an important and necessary step towards clarifying the effect of taurine on diabetic hypercholesterolemia.

In the present study, we examined the effect of taurine on the cholesterol metabolism in genetic diabetic GK rats fed diets supplemented with and without cholesterol. GK rats are genetic type 2 diabetic rats produced by the selective breeding of normal Wistar rats by Goto et al. (9). This rat does not show obesity and secretes insufficient amounts of insulin in response to glucose feeding. The animal also has high plasma insulin levels and is insulin resistant (10).

\section{MATERIALS AND METHODS}

Animals and diets. Male Wistar rats $(100 \mathrm{~g})$ and male GK rats $(100 \mathrm{~g})$ were used (Japan Clea, Tokyo, 
Japan) as the normal and diabetic rats, respectively. The rats were housed in individual stainless steel cages with screen bottoms in a room maintained at $23 \pm 1^{\circ} \mathrm{C}$ with lighting from 0700 to 1900 . The rats were acclimated by feeding a $20 \%$ casein diet free of cholesterol for $3 \mathrm{~d}$. The composition of the cholesterol-free diet is shown in Table 1. Taurine was supplied from Taisho Pharmaceutical Co., Ltd. (Tokyo, Japan).

After the acclimation period, the rats were divided into two groups of six each based on their body weight and plasma cholesterol concentration, and were administered test diets for $21 \mathrm{~d}$ (experiment 1 ) or $14 \mathrm{~d}$ (experiment 2). In the present study, four test groups were used in a $2 \times 2$ design, using normal or diabetic rats and taurine-free or taurine-supplemented diets. The compositions of the experimental diets are shown in Table 1. Blood samples $(50 \mu \mathrm{L})$ were collected from the tail veins at $1000-1100 \mathrm{~h}$ on day $0,3,7,14$, and 21 (experiment 1 ) or $0,3,7$, and 14 (experiment 2) for determination of the total plasma cholesterol concentration and blood glucose concentration, respectively.

The study was approved by the University of Shizuoka Animal Use Committee, and the animals were maintained in accordance with the guidelines for the care and use of laboratory animals, University of Shizuoka.

Sampling procedures. At the end of the experimental period, the rats were sacrificed by decapitation at 1300-1400 h, and blood was removed via a funnel into a centrifuging tube containing heparin. The plasma was separated by centrifugation at $1,200 \times g$ for $20 \mathrm{~min}$ at $4^{\circ} \mathrm{C}$. The liver was removed, weighed, immediately frozen in liquid nitrogen, and stored at $-40^{\circ} \mathrm{C}$ until analysis of the cholesterol.

For the determination of the total bile acids excreted into the feces, the feces were collected during the last $3 \mathrm{~d}$ of the experimental period, lyophilized, weighed, and stored at $-40^{\circ} \mathrm{C}$.

Biochemical analyses. The plasma cholesterol concentration was determined by an enzymatic method using a commercial kit (Cholesterol C-test, Wako, Osaka, Japan). The plasma (VLDL+LDL) fraction was precipitated with a heparin:manganese reagent (Wako) and the supernatant (the HDL fraction) was assayed for HDL-cholesterol using a commercial kit (HDL-cholesterol test, Wako). The concentration of (VLDL+LDL) cholesterol was calculated as the difference between the total plasma cholesterol concentration and the HDL cholesterol concentration. The plasma triglyceride and phospholipid concentrations were measured using commercial kits (Triglyceride $G$ test and Phospholipid B test, respectively, Wako). The blood and plasma glucose concentrations were analyzed by commercial kits (TIDEX, Bayer Medical, Tokyo, Japan and Glucose CII test, Wako, respectively). The plasma insulin concentration was measured with an antibody-sandwich ELISA method using a "Lbis Rat Insulin" kit (Shibayagi, Gunma, Japan). The rat insulin supplied with the kit was used as the standard. The determination range was $0.156-10 \mathrm{ng} / \mathrm{mL}$.
Table 1. Composition of the experimental diets.

\begin{tabular}{|c|c|c|c|c|}
\hline & \multicolumn{2}{|c|}{ Cholesterol-free } & \multicolumn{2}{|c|}{ High cholesterol } \\
\hline & Control & Taurine & Control & Taurine \\
\hline & \multicolumn{4}{|c|}{$\mathrm{g} / \mathrm{kg}$} \\
\hline Casein & 200 & 200 & 200 & 200 \\
\hline$\alpha$-Cornstarch & 435.7 & 415.7 & 427.4 & 407.4 \\
\hline Sucrose & 217.8 & 207.8 & 213.6 & 203.6 \\
\hline Mineral mix. ${ }^{1}$ & 35 & 35 & 35 & 35 \\
\hline Vitamin mix. $^{1}$ & 10 & 10 & 10 & 10 \\
\hline Corn oil & 5 & 5 & 5 & 5 \\
\hline Cellulose & 5 & 5 & 5 & 5 \\
\hline Choline chloride & 1.5 & 1.5 & 1.5 & 1.5 \\
\hline Cholesterol & - & - & 10 & 10 \\
\hline Sodium cholate & - & - & 2.5 & 2.5 \\
\hline Taurine & - & 30 & - & 30 \\
\hline
\end{tabular}

${ }^{1}$ AIN-93 (11).

The liver cholesterol was analyzed with a commercial kit (Cholesterol $\mathrm{C}$ test) using the method of Morita et al. (12) after extraction of the lipid from the liver by the method of Folch et al. (13). The total fecal bile acids were extracted from the lyophilized feces using a mixture of chloroform-methanol $(1: 1, \mathrm{v} / \mathrm{v})$ at $70^{\circ} \mathrm{C}$ for $60 \mathrm{~h} \mathrm{(14)}$ and enzymatically analyzed by the $3 \alpha$-hydroxysteroid dehydrogenase assay of Sheltaway and Lowosky (15) with slight modification. Lithocholic acid was used as the standard substrate.

Statistical analysis. The experimental data were statistically analyzed by two-way ANOVA (16). When the interaction (diabetes $\times$ taurine) was significant $(p<0.05)$, Student's $t$-test was performed (17). All the statistical analyses were performed using the Statistical Analysis System (SAS/STAT Version 6, SAS Institute, Cary, NC, USA).

\section{RESULTS}

Experiment 1 (experiment with cholesterol-free diets)

The body weight gains of normal rats fed the control and taurine diets $(168 \pm 2$ and $167 \pm 4 \mathrm{~g} / 21 \mathrm{~d})$ were significantly higher compared to the diabetic rats fed the control and taurine diets $(108 \pm 7$ and $115 \pm 3 \mathrm{~g} / 21 \mathrm{~d}$, ANOVA analysis, $p<0.001$ ). Food intake was significantly higher in the normal rats fed the control and taurine diets $(388 \pm 4$ and $397 \pm 9 \mathrm{~g} / 21 \mathrm{~d})$ than in the diabetic rats fed the control and taurine diets $(268 \pm 12$ and $284 \pm 6 \mathrm{~g} / 21 \mathrm{~d}$, ANOVA analysis, $p<0.001$ ).

Figure 1 shows the time-dependent changes in the plasma cholesterol and blood glucose concentrations in rats fed the cholesterol-free diets supplemented with or without taurine. On day 0 , a significantly high plasma cholesterol concentration was observed in the diabetic rats as compared to the normal rats (ANOVA analysis, $p<0.001$ ). For the diabetic rats, the plasma cholesterol concentration was significantly higher in the taurinefed rats than in those fed the taurine-free diet at 14 and $21 \mathrm{~d}$ of the experiment $(p<0.05$ and $p<0.001$, respectively), and there was no difference as the result of tau- 


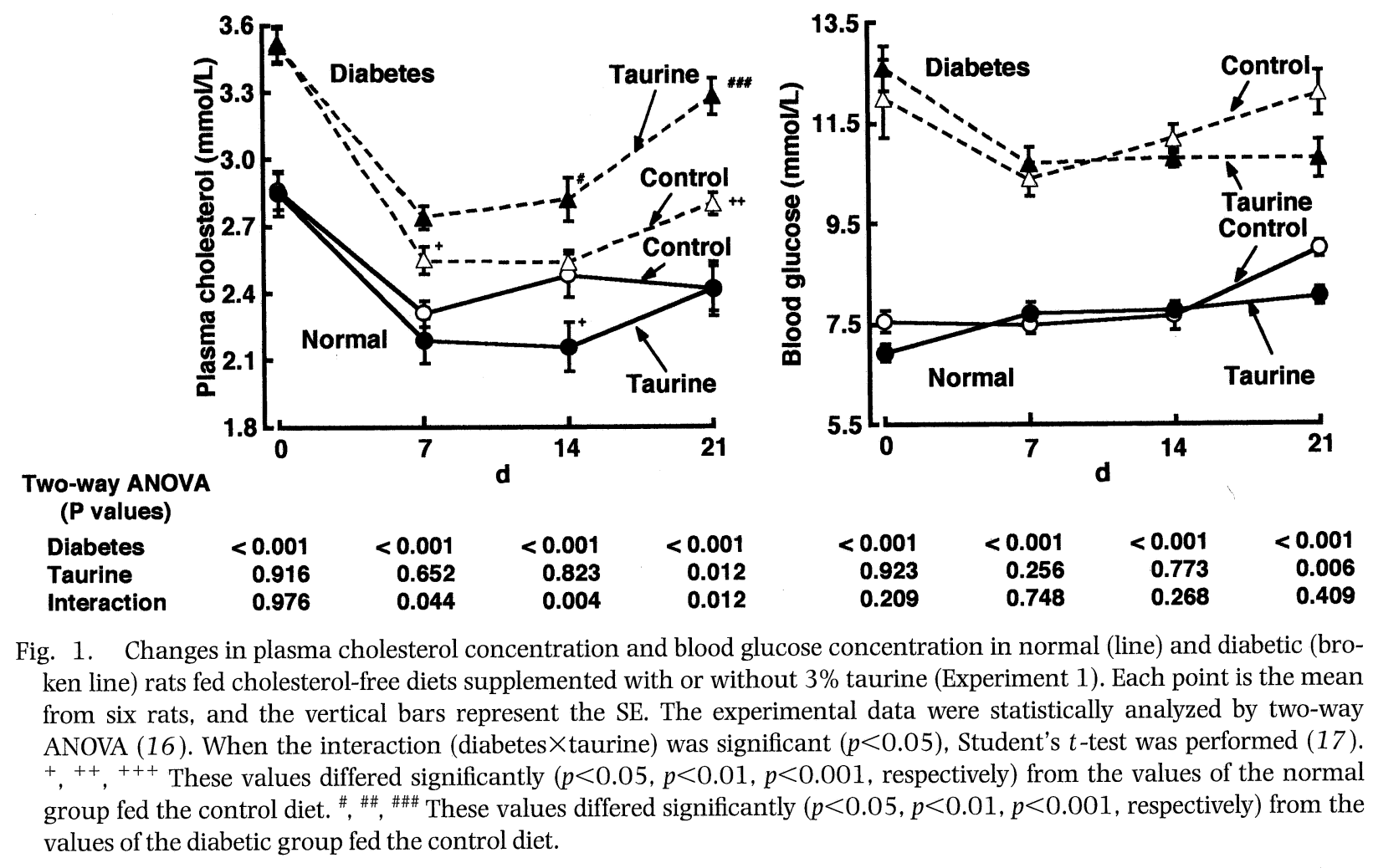

rine treatment in the normal rats, except at $14 \mathrm{~d}$. The blood glucose concentration remained high in the diabetic rats throughout the experimental period as compared to the normal rats, and was not affected by taurine administration except on day 21.

Table 2 shows the plasma concentrations of lipids, glucose, and insulin in the rats fed the cholesterol-free diets at $21 \mathrm{~d}$ of the experiment. The total plasma cholesterol concentration was significantly higher in the diabetic rats as compared to the normal rats (ANOVA analysis, $p<0.001$ ). When taurine was administered to the diabetic rats, the plasma cholesterol concentration increased $(p<0.01)$, and this rise was due to the increase in plasma HDL-cholesterol. The plasma triglyceride concentration was significantly lower in the diabetic rats than in the normal rats (ANOVA analysis, $p<0.001$ ), and was significantly reduced by taurine feeding only in the normal rats $(p<0.001)$. The plasma phospholipid concentration was higher in the diabetic rats than in the normal rats (ANOVA analysis, $p<0.001$ ). The plasma phospholipids were significantly higher in the diabetic rats fed taurine than in those fed the control diet $(p<0.05)$. The plasma glucose and insulin levels were significantly higher in the diabetic rats than in the normal rats (ANOVA analysis, $p<0.001$ ). However, the plasma glucose and insulin levels in the taurine-fed rats were not significantly different from the levels in the rats fed the taurine-free diet, except for the plasma glucose in the diabetic rats. No significant difference in liver cholesterol concentration was observed between the rats fed the taurine-free diets and taurine diets in both the normal and diabetic rats (Table 3 ).

The daily excretion of fecal bile acids was significantly greater in the normal rats than in the diabetic rats
(ANOVA analysis, $p<0.001$ ), and was significantly enhanced by the feeding of taurine (ANOVA analysis, $p=0.035$, Table 3 ).

Experiment 2 (experiment with high cholesterol diets)

The body weight gains were significantly higher in the normal rats fed the control and taurine diets $(104 \pm 3$ and $105 \pm 4 \mathrm{~g} / 14 \mathrm{~d})$ than in the diabetic rats fed respective diets $(69 \pm 3$ and $72 \pm 3 \mathrm{~g} / 14 \mathrm{~d}$, ANOVA analysis, $p<0.001$ ). The food intake of the normal rats fed the control and taurine diets $(266 \pm 3$ and $279 \pm$ $4 \mathrm{~g} / 14 \mathrm{~d}$ ) was significantly higher than that of the diabetic rats fed the control and taurine diets $(204 \pm 4$ and $216 \pm 4 \mathrm{~g} / 14 \mathrm{~d}$, ANOVA analysis, $p<0.001$ ).

Figure 2 shows the time-dependent changes in the plasma cholesterol and blood glucose concentrations in the rats fed the high-cholesterol diets supplemented with or without taurine. On day 0 , the plasma cholesterol concentration was significantly higher in the diabetic rats than in the normal rats (ANOVA analysis, $p<0.001)$. The low plasma cholesterol concentration was maintained in the diabetic rats fed the control diet supplemented with cholesterol throughout the experimental period. At 7 and $14 \mathrm{~d}$, the plasma cholesterol concentration was significantly lower in the taurine-fed rats than in the rats fed the control diet (ANOVA analysis, $p<0.001$ ). Compared to the normal rats, the blood glucose concentration was higher in the diabetic rats throughout the experimental period (ANOVA analysis, $p<0.001$ ). The blood glucose concentration did not differ between the control groups and taurine-fed groups for both the normal rats and diabetic rats.

Table 4 shows the plasma parameters for the lipids, glucose, and insulin in the cholesterol-fed rats on day 14 of the experiment. The total and (VLDL+LDL) cho- 


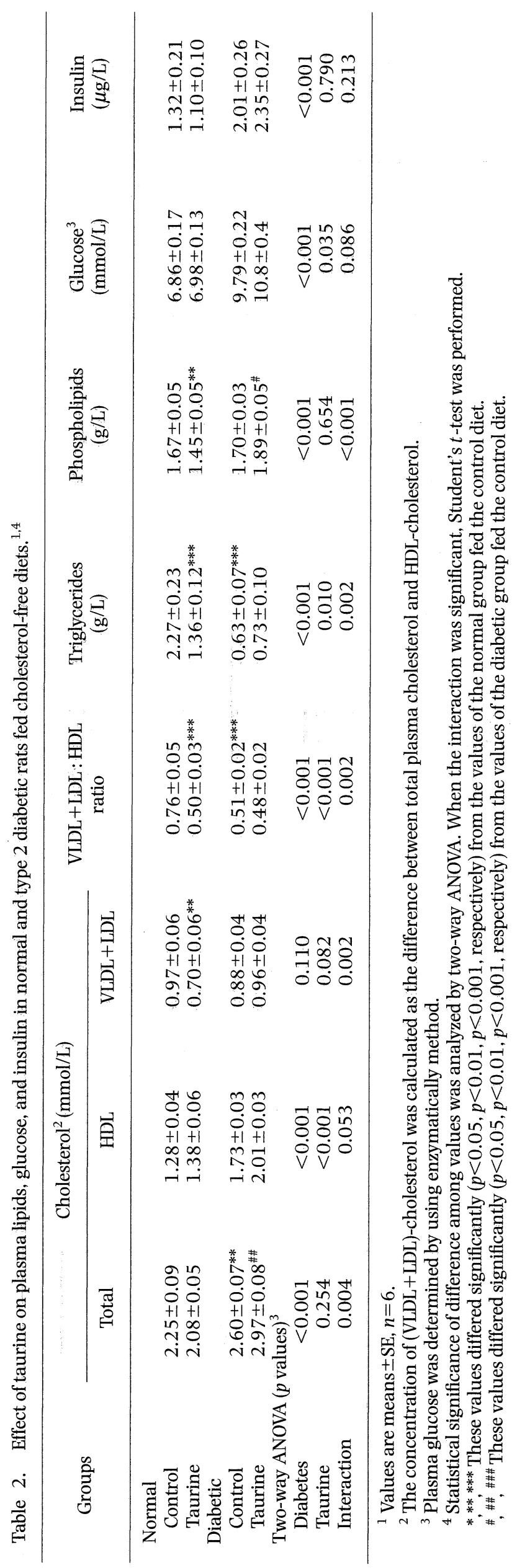

lesterol concentrations in plasma were significantly greater in the normal rats fed the control diet than in the diabetic rats fed the same diet (ANOVA analysis, $p<0.001$ ). The total and (VLDL+LDL) cholesterol concentrations in the plasma were significantly lower in the rats fed the taurine diet than in those fed the control $\operatorname{diet}$ (ANOVA analysis, $p<0.001$ ). The plasma HDL-cholesterol concentration was significantly higher in the diabetic rats than in the normal rats (ANOVA analysis, $p<0.001$ ). Taurine feeding significantly increased the HDL-cholesterol concentration in the diabetic rats but not in normal rats. The plasma insulin was significantly higher in the diabetic rats than in the normal rats (ANOVA analysis, $p<0.001$ ). The daily excretion of fecal bile acids was significantly higher in the normal rats than in the diabetic rats (ANOVA analysis, $p<0.001$, Table 5), and in the taurine-fed rats than in rats fed the control diet (ANOVA analysis, $p<0.001$, Table 5).

\section{DISCUSSION}

In the present study, at the start of the experiment, a high plasma cholesterol concentration was observed in the diabetic rats as compared to that of the normal rats, suggesting that the change in cholesterol metabolism was induced by the development of diabetes mellitus. In the diabetic rats, taurine feeding increased the HDLcholesterol, and also decreased the (VLDL+LDL)cholesterol concentration in the cholesterol-fed rats. From these results, it is suggested that taurine affects the cholesterol metabolism in diabetic rats. In a previous study, we found that taurine increased the HDL-cholesterol concentration in normal rats (6) and polychlorinated biphenyls-fed rats (18). We also proved that taurine lowered the VLDL-cholesterol concentration in cholesterol-fed rats (5).

The present and previous studies $(7,8)$ show that taurine affects the plasma cholesterol concentration when the lipid metabolism is changed by diabetes mellitus. Luoma et al. reported that an alternation in the lipoprotein composition in the serum was observed in humans with NIDDM (19). It is well known that the elevation of serum triglyceride (VLDL) and reduced HDL characterize the lipid profile in $\operatorname{NIDDM}(2,3,20)$. To improve the lipoprotein profiles in serum by taurine intake seems to be effective for preventing diabetic hypercholesterolemia.

The plasma cholesterol in diabetic GK rats fed the cholesterol-free diet was significantly elevated by taurine feeding. This elevation depended on the HDL-fraction. We previously observed that taurine intake raised the HDL-fraction in the serum in normal rats fed a cholesterol-free diet (6). Because apo A-I is the principal apolipoprotein consisting of HDL, the content of apo A-I may be related to the formation of HDL in the liver and the concentration of serum HDL-cholesterol (21). In our previous study, the concentration of hepatic apo A-I mRNA tended to be greater in taurine-fed rats (6). Therefore, in addition to normal rats, HDL-cholesterol concentration might be elevated through inducing ex- 
Table 3. Effect of taurine on liver weight and cholesterol, and fecal bile acid excretion in normal and type 2 diabetic rats fed cholesterol-free diets. ${ }^{1}$

\begin{tabular}{|c|c|c|c|c|}
\hline \multirow{2}{*}{ Groups } & \multicolumn{2}{|c|}{ Liver } & \multicolumn{2}{|c|}{ Feces } \\
\hline & $\begin{array}{c}\text { Weight } \\
\text { (g/100 g body wt.) }\end{array}$ & $\begin{array}{l}\text { Cholesterol } \\
(\mu \mathrm{mol} / \mathrm{g} \text { liver })\end{array}$ & $\begin{array}{l}\text { Weight } \\
(\mathrm{g} / \mathrm{d})\end{array}$ & $\begin{array}{l}\text { Bile acids } \\
(\mu \mathrm{mol} / \mathrm{d})\end{array}$ \\
\hline \multicolumn{5}{|l|}{ Normal } \\
\hline Control & $4.03 \pm 0.04$ & $6.99 \pm 0.08$ & $1.80 \pm 0.06$ & $13.4 \pm 3.4$ \\
\hline Taurine & $3.89 \pm 0.05$ & $6.93 \pm 0.17$ & $1.82 \pm 0.06$ & $18.0 \pm 1.0$ \\
\hline \multicolumn{5}{|l|}{ Diabetic } \\
\hline Control & $4.11 \pm 0.09$ & $6.82 \pm 0.16$ & $1.17 \pm 0.07$ & $7.8 \pm 1.0$ \\
\hline Taurine & $4.25 \pm 0.07$ & $6.46 \pm 0.11$ & $1.31 \pm 0.08$ & $11.6 \pm 0.6$ \\
\hline \multicolumn{5}{|c|}{ Two-way ANOVA ( $p$ values) ${ }^{2}$} \\
\hline Diabetes & 0.003 & 0.030 & $<0.001$ & $<0.001$ \\
\hline Taurine & 0.931 & 0.139 & 0.263 & 0.035 \\
\hline Interaction & 0.051 & 0.268 & 0.400 & 0.842 \\
\hline
\end{tabular}

\footnotetext{
${ }^{1}$ Values are means \pm SE, $n=6$.

${ }^{2}$ Statistical significance of difference among values was analyzed by two-way ANOVA. When the interaction was significant, Student's $t$-test was performed.
}
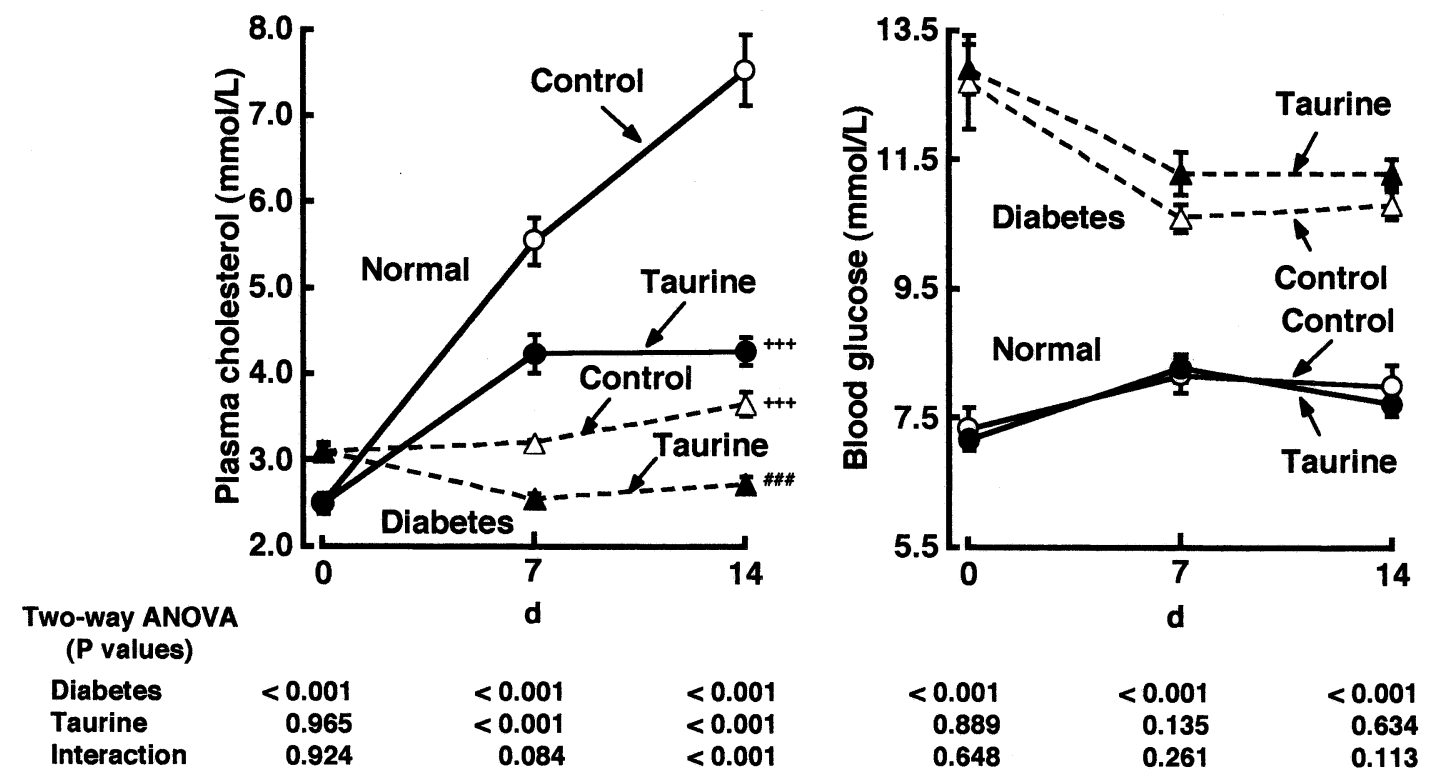

Fig. 2. Changes in plasma cholesterol concentration and blood glucose concentration in normal (line) and diabetic (broken line) rats fed high-cholesterol diets supplemented with or without 3\% taurine (Experiment 2). Each point is the mean from six rats, and the vertical bars represent the SE. The experimental data were statistically analyzed by two-way ANOVA (16). When the interaction (diabetes $\times$ taurine) was significant $(p<0.05)$, Student's $t$-test was performed (17). ${ }^{+},{ }^{++},{ }^{+++}$These values differed significantly $(p<0.05, p<0.01, p<0.001$, respectively) from the values of the normal group fed the control diet. \#, \#, \#\#\# These values differed significantly $(p<0.05, p<0.01, p<0.001$, respectively) from the values of the diabetic group fed the control diet.

pression of the hepatic apo A-I gene by feeding taurine to type 2 diabetic rats fed a cholesterol-free as well. The plasma cholesterol fell from day 0 to 7 in all the groups fed the cholesterol-free diets. It is not clear how this phenomenon occurs. However, rats were not subjected to any stress as part of the experimental design and showed no visible signs of stress.

It is well known that CYP7A1 contributes to the regulation of serum cholesterol concentration, especially (VLDL+LDL)-cholesterol concentration (5, 22). However, there is little agreement on the change in hepatic CYP7A1 activity in diabetes, especially type 2 diabetes. CYP7A1 is an insulin-sensitive enzyme, and its activity is suppressed by insulin (23). In the present study, the plasma insulin was higher in the diabetic rats. Fecal bile acid excretion, which positively correlates with hepatic CYP7A1 activity (24), was lower in the type 2 diabetic rats than in normal rats. A high level of plasma insulin from diabetes might suppress the hepatic CYP7A1 activity. We previously reported that the reduction of (VLDL+LDL)-cholesterol through taurine intake was due to the enhancement of hepatic CYP7A1 activity and its gene expression (5). Therefore, taurine may normalize the high (VLDL+LDL)-cholesterol con- 
centration in type 2 diabetic rats as well as normal rats by promoting CYP7A1 activity, independent of the effect of high plasma insulin levels. However, it is unclear whether or not CYP7A1 activity is independent of plasma insulin concentration in type 2 diabetes.

In the present study, high glucose concentrations (ca. $11 \mathrm{mmol} / \mathrm{L}$ ) in the plasma of diabetic rats were maintained, but the plasma insulin concentrations were higher in diabetic rats than in normal rats. Also, when the rats were sacrificed, the digesta did not remain in the stomach. Therefore, the difference in the plasma glucose and insulin concentration in the normal and diabetic rats seems not to be dependent on different feeding patterns. From these data, it is presumed that the diabetic rats were insulin resistant. The GK rat is a spontaneous non-obese model for type 2 diabetes (9). The animal has high levels of plasma glucose and insulin and shows insulin resistance (10). In the present study, total and (VLDL+LDL)-cholesterol concentrations, as well as triglycerides in the plasma, were lower in cholesterol-fed diabetic rats as compared to normal rats fed cholesterol. Additionally, compared to normal rats, an increase in the plasma HDL-cholesterol was observed in diabetic rats. These results are opposite of those reported in other research on type 2 diabetes $(2$, 3, 20).

An accumulation of cholesterol in the liver was observed in the cholesterol-fed diabetic rats. Therefore, cholesterol absorption from the small intestine in diabetic rats was surely unaffected compared to that of the normal rats. This different response may reflect the peculiar metabolism of cholesterol in type 2 diabetic GK rats.

We previously observed that the VLDL fraction in serum lipoprotein was notably increased by cholesterol intake in normal and streptozotocin-induced diabetic rats $(5,8)$, but not in GK rats in the present study. Compared to normal rats, the ability to release VLDL from the liver may be low, or the catabolism of VLDL fast, in GK rats. However, VLDL release is stimulated by insulin (20), so lower (VLDL+LDL)-cholesterol in GK rats fed cholesterol seems not to be due to a change in VLDL release.

Insulin also promotes the activity of lipoprotein lipase $(20,25)$. Therefore, the catabolism of VLDL may be faster in diabetic GK rats (high insulin) than in normal rats (normal insulin). The Otsuka Long-Evans Tokushima Fatty (OLETF) rat is also a type 2 diabetes model with high insulin and insulin resistance, but is obese. Nakaya et al. (26) reported that plasma cholesterol and triglyceride are increased by cholesterol intake in OLETF rats. In the present study and the study of Nakaya et al. (26), taurine feeding significantly decreased plasma (VLDL+LDL)-cholesterol. In our unpublished data, taurine suppressed VLDL release from the liver in cholesterol-fed normal rats administered Triton WR1339. Therefore, taurine may be involved in the inhibition of VLDL release and/or the promotion of VLDL degradation.

Our results indicate that taurine has a plasma choles- 
Table 5. Effect of taurine on liver weight and cholesterol, and fecal bile acid excretion in normal and type 2 diabetic rats fed high cholesterol diets. ${ }^{1}$

\begin{tabular}{|c|c|c|c|c|}
\hline \multirow{2}{*}{ Groups } & \multicolumn{2}{|c|}{ Liver } & \multicolumn{2}{|c|}{ Feces } \\
\hline & $\begin{array}{c}\text { Weight } \\
\text { (g/100 g body wt.) }\end{array}$ & $\begin{array}{l}\text { Cholesterol } \\
(\mu \mathrm{mol} / \mathrm{g} \text { liver })\end{array}$ & $\begin{array}{l}\text { Weight } \\
(\mathrm{g} / \mathrm{d})\end{array}$ & $\begin{array}{l}\text { Bile acids } \\
(\mu \mathrm{mol} / \mathrm{d})\end{array}$ \\
\hline \multicolumn{5}{|l|}{ Normal } \\
\hline Control & $6.35 \pm 0.15$ & $161.2 \pm 6.1$ & $1.79 \pm 0.05$ & $140.0 \pm 5.2$ \\
\hline Taurine & $5.94 \pm 0.10$ & $98.2 \pm 5.5$ & $2.12 \pm 0.10$ & $164.2 \pm 10.1$ \\
\hline \multicolumn{5}{|l|}{ Diabetic } \\
\hline Control & $6.13 \pm 0.19$ & $182.9 \pm 8.9$ & $1.44 \pm 0.04$ & $86.8 \pm 5.2$ \\
\hline Taurine & $6.27 \pm 0.17$ & $133.0 \pm 6.8$ & $1.63 \pm 0.08$ & $123.4 \pm 5.0$ \\
\hline \multicolumn{5}{|c|}{ Two-way ANOVA ( $p$ values) ${ }^{2}$} \\
\hline Diabetes & 0.725 & $<0.001$ & $<0.001$ & $<0.001$ \\
\hline Taurine & 0.409 & $<0.001$ & 0.002 & $<0.001$ \\
\hline Interaction & 0.086 & 0.352 & 0.372 & 0.373 \\
\hline
\end{tabular}

\footnotetext{
${ }^{1}$ Values are means \pm SE, $n=6$. Student's $t$-test was performed.

terol-lowering effect in genetic type 2 diabetic rats fed high-cholesterol diets as well as in normal rats, as reflected in the reduction of the (VLDL+LDL) fraction. It is suggested that taurine also affects the HDL-cholesterol metabolism in genetic type 2 diabetic rats.
}

${ }^{2}$ Statistical significance of difference among values was analyzed by two-way ANOVA. When the interaction was significant,

\section{Acknowledgments}

This study was supported by a grant from Taisho Pharmaceutical Co., Ltd., Tokyo, Japan.

\section{REFERENCES}

1) Steiner G. 1978. Hyperlipidemia, atherosclerosis, and diabetes. Prim Care 5: 81-89.

2) Howard BV. 1987. Lipoprotein metabolism in diabetes mellitus. J Lipid Res 28: 613-628.

3) Nikkila EA, Huttunen JK, Ehnholm C. 1977. Postheparin plasma lipoprotein lipase and hepatic lipase in diabetes mellitus. Relationship to plasma triglyceride metabolism. Diabetes 26: 11-21.

4) Tavangar K, Murata Y, Pedersen ME, Goers JF, Hoffman AR, Kraemer FB, Chan CP, Stern JS. 1992. Regulation of lipoprotein lipase in the diabetic rat. J Clin Invest 90: 1672-1678.

5) Yokogoshi H, Mochizuki H, Nanami K, Hida Y, Miyachi F, Oda H. 1999. Dietary taurine enhances cholesterol degradation and reduces plasma and liver cholesterol concentrations in rats fed a high-cholesterol diet. J Nutr 129: 1705-1712.

6) Mochizuki H, Oda H, Yokogoshi H. 1998. Increasing effect of dietary taurine on the serum HDL-cholesterol concentration in rats. Biosci Biotechnol Biochem 62: 578-579.

7) Nanami K, Oda H, Yokogoshi H. 1996. Antihypercholesterolemic action of taurine on streptozotocin-diabetic rats or on rats fed a high cholesterol diet. In: Taurine 2 (Huxtable RJ et al., eds), p 561-568. Plenum Press, New York.

8) Mochizuki H, Takido J, Oda H, Yokogoshi H. 1999. Improving effect of dietary taurine on marked hypercholesterolemia induced by a high-cholesterol diet in streptozotocin-induced diabetic rats. Biosci Biotechnol Biochem 63: 1984-1987.

9) Goto Y, Kakizaki M, Masaki N. 1975. Spontaneous diabetes produced by selective breeding of normal Wistar rats. Proc Jpn Acad 51: 80-85.

10) Ostenson CG, Khan A, Abdel-Halim SM, Guenifi A, Suzuki K, Goto Y, Efendic S. 1993. Abnormal insulin secretion and glucose metabolism in pancreatic islets from the spontaneously diabetic GK rat. Diabetologia 36: 3-8.

11) Reeves PG, Nielsen FH, Fahey GC Jr. 1993. AIN-93 purified diets for laboratory rodents: final report of the American Institute of Nutrition Ad Hoc Writing Committee on the reformulation of the AIN-76A rodent diet. J Nutr 123: 1939-1951.

12) Morita T, Oh-hashi A, Takei K, Ikai M, Kasaoka S, Kiriyama S. 1997. Cholesterol-lowering effects of soybean, potato and rice proteins depend on their low methionine contents in rats fed a cholesterol-free purified diet. J Nutr 127: 470-477.

13) Folch J, Less M, Sloane-Stanley GH. 1957. A simple method for the isolation and purification of total lipids from animal tissues. J Biol Chem 226: 497-509.

14) Eneroth P, Hellstrom K, Sjovall J. 1968. A method for quantitative determination of bile acids in human feces. Bile acids and steroids 195. Acta Chem Scand 22: 1729-1744.

15) Sheltaway MJ, Lowosky MS. 1975. Determination of fecal bile acids by an enzymatic method. Clin Chim Acta 64: 127-132.

16) Oda H, Fukui H, Hitomi Y, Yoshida A. 1991. Alternation of serum lipoprotein metabolism by polychlorinated biphenyls and methionine in rats fed a soybean protein diet. J Nutr 121: 925-933.

17) Snedecor GW, Cochran WG. 1967. Statistical Methods, 6th ed. Iowa State University Press, Ames, IA.

18) Mochizuki H, Oda H, Yokogoshi H. 2001. Dietary taurine potentiates polychlorinated biphenyl-induced hypercholesterolemia in rats. $J$ Nutr Biochem 12: 109-115.

19) Luoma PV, Savolainen MJ, Sotaniemi EA, Arranto AJ, 
Pelkonen RO. 1985. Plasma HDL cholesterol and blood glucose in non-insulin dependent diabetics related to liver lipids and microsomal enzyme activity. Acta Med Scand 21 7: 473-479.

20) Taskinen MR. 1990. Diabetes, hypertriglyceridaemia and high density lipoprotein. In: Disorders of HDL-Selected Proceedings (Carlson LA, ed), p 35-42. SmithGordon, London.

21) Bamberger MJ, Lane MD. 1988. Assembly of very low density lipoprotein in the hepatocyte. Differential transport of apoproteins through the secretory pathway. $J$ Biol Chem 263: 11868-11878.

22) Horton JD, Cuthbert JA, Spady DK. 1995. Regulation of hepatic $7 \alpha$-hydroxylase expression and response to dietary cholesterol in the rat and hamster. J Biol Chem
270: $5381-5387$.

23) Subbiah MT, Yunker RL. 1984. Cholesterol $7 \alpha$-hydroxylase of rat liver: an insulin sensitive enzyme. Biochem Biophys Res Commun 124: 896-902.

24) Turley SD, Schwarz M, Spady DK, Dietschy JM. 1998. Gender-related differences in bile acid and sterol metabolism in outbred CD-1 mice fed low- and high-cholesterol diets. Hepatology 28: 1088-1094.

25) Taskinen MR. 1987. Lipoprotein lipase in diabetes. Diabetes Metab Rev 3: 551-570.

26) Nakaya Y, Minami A, Harada N, Sakamoto S, Niwa Y, Ohnaka M. 2000. Taurine improves insulin sensitivity in the Otsuka Long-Evans Tokushima Fatty rat, a model of spontaneous type 2 diabetes. Am J Clin Nutr $\mathbf{7 1}$ : 54-58. 